US-FT/22-95

\title{
A-DEPENDENCE OF HEAVY FLAVOUR PRODUCTION IN QCD
}

\author{
N.Armesto, C.Pajares, C.A.Salgado and Yu.M.Shabelski* \\ Departamento de Fisica de Partículas, Universidade de Santiago de Compostela, \\ 15706-Santiago de Compostela, Spain
}

\begin{abstract}
We calculate the A-dependence of charm and beauty production cross sections on nuclear targets taking into account the difference of quark and gluon distributions in free nucleons and in nucleus. At comparatively low energies, if $\sigma \sim A^{\alpha}$, the value of $\alpha$ is slightly higher than unity. With the growth of the initial energy the value of $\alpha$ decreases and becomes smaller than unity. We also calculate the values of $\alpha$ for different Feynman- $x$ of the produced $Q \bar{Q}$ pair and obtain that they decrease significantly in the beam fragmentation region.
\end{abstract}

*) Permanent address: Petersburg Nuclear Physics Institute,

Gatchina, Sanct-Petersburg 188350, Russia.

August 1995

US-FT/22-95 


\section{Introduction}

The processes of heavy flavour production on nucleon and nucleus targets at high energies are very interesting from both the theoretical and practical points of view. These processes at high energies are usually considered in the framework of perturbative QCD in the leading and the next-to-leading order $\alpha_{s}$ expansion. In the case of a nuclear target it is usually assumed that the cross section of heavy flavour production, $\sigma(Q \bar{Q})$, should be proportional to $A^{\alpha}$, with $\alpha=1$, that is, in agreement with the experimental result of [1], where a value $\alpha=1.02 \pm 0.03 \pm 0.02$ was obtained for the case of neutral D-meson production at $\sqrt{s}=39 \mathrm{GeV}$. However it is experimentally well-known [2-8] that quark distributions in nucleus are slightly different from the same distributions in free nucleons. So the value of $\alpha$ can differ from unity and it seems interesting to estimate it. We will present in the Section 2 the results obtained using the nucleus to nucleon structure function ratios taken from Ref. [9].

\section{QCD predictions of charm and beauty production A-dependence}

The standard QCD expression for heavy quark production cross section in a hadron 1 hadron 2 collision has the form

$$
\sigma^{12 \rightarrow Q \bar{Q}}=\int_{x_{a 0}}^{1} \frac{d x_{a}}{x_{a}} \int_{x_{b 0}}^{1} \frac{d x_{b}}{x_{b}}\left[x_{a} G_{a / 1}\left(x_{a}, \mu^{2}\right)\right]\left[x_{b} G_{b / 2}\left(x_{b}, \mu^{2}\right)\right] \hat{\sigma}^{a b \rightarrow Q \bar{Q}}\left(\hat{s}, m_{Q}, \mu^{2}\right)
$$

where $x_{a 0}=4 m_{Q}^{2} / s$ and $x_{b 0}=4 m_{Q}^{2} /\left(s x_{a}\right)$. Here $G_{a / 1}\left(x_{a}, \mu^{2}\right)$ and $G_{b / 2}\left(x_{b}, \mu^{2}\right)$ are the structure functions of partons $a$ and $b$ inside hadrons 1 and 2 respectively, and $\hat{\sigma}^{a b \rightarrow Q \bar{Q}}\left(\hat{s}, m_{Q}, \mu^{2}\right)$ is the cross section of the subprocess $a b \rightarrow Q \bar{Q}$ as given by standard QCD. The latter depends on the parton center-of-mass energy $\hat{s}=\left(p_{a}+p_{b}\right)^{2}=x_{a} x_{b} s$, the mass of the produced heavy quark $m_{Q}$ and the QCD scale $\mu^{2}$. Eq. (1) should account for all possible subprocesses $a b \rightarrow Q \bar{Q}$.

The parton cross section $\hat{\sigma}^{a b \rightarrow Q \bar{Q}}\left(\hat{s}, m_{Q}, \mu^{2}\right)$ can be written in the form 10

$$
\hat{\sigma}^{a b \rightarrow Q \bar{Q}}\left(\hat{s}, m_{Q}, \mu^{2}\right)=\frac{\alpha_{s}^{2}\left(\mu^{2}\right)}{m_{Q}^{2}} f_{a b}\left(\rho, \mu^{2} / m_{Q}^{2}\right),
$$

with

$$
\rho=4 m_{Q}^{2} / \hat{s}
$$

and

$$
f_{a b}\left(\rho, \mu^{2} / m_{Q}^{2}\right)=f_{a b}^{(0)}(\rho)+4 \pi \alpha_{s}\left(\mu^{2}\right)\left[f_{a b}^{(1)}(\rho)+\hat{f}_{a b}^{(1)}(\rho) \ln \left(\mu^{2} / m_{Q}^{2}\right)\right] .
$$


The functions $f_{a b}^{(0)}(\rho), f_{a b}^{(1)}(\rho)$ and $\hat{f}_{a b}^{(1)}(\rho)$ can be found in [10].

For the numerical calculations we wrote the nuclear structure function $G_{b / A}\left(x_{b}, \mu^{2}\right)$ in the form

$$
G_{b / A}\left(x_{b}, \mu^{2}\right)=A \cdot G_{b / N}\left(x_{b}, \mu^{2}\right) \cdot R_{b}^{A}\left(x, \mu^{2}\right),
$$

similarly to Ref. [11] and we take the values of $R_{b}^{A}\left(x, \mu^{2}\right)$ for gluons, valence and sea quarks from Ref. [9] They are presented in Fig. 1. The values of $R_{b}^{A}\left(x, \mu^{2}\right)$ in [9] are presented for $x>10^{-3}$ that is not small enough at high energies. So at $x<10^{-3}$ we used two variants of the $R_{b}^{A}\left(x, \mu^{2}\right)$ behaviour for gluon distributions: The first is the extrapolation as $x^{\beta}$ with $\beta=0.096$ and 0.040 for the charm and beauty production, respectively (solid lines in Fig. 1). Such behaviour is in qualitative agreement with the results of [13]. The second is the constant frozen at $x=10^{-3}$ (dash-dotted lines). Three different sets of parton distributions were used, namely MRS-1 [14], MT S-DIS [15] and GRV HO [16] that can be found in CERN PDFLIB [17], but they give practically the same results for the $\alpha$ behaviour (see Table 1). We have used in the case of charm production the values $m_{c}=1.5 \mathrm{GeV}$ and $\mu^{2}=4 \mathrm{GeV}^{2}$ and in the case of beauty production $m_{b}=$ $5 \mathrm{GeV}$ and $\mu^{2}=m_{b}^{2}$.

The obtained results for $\alpha$ determined from the ratios of heavy quark production cross section on a gold target and on the proton are presented in the Table 1 for three sets of structure functions and frozen gluon distribution ratios at $x=10^{-3}$ and in Fig. 2 for the GRV HO set and two variants of gluon distribution ratios in the small $x$ region. Here $\sqrt{s_{N N}}$ is the c.m. energy for the interaction of the incident proton with one target nucleon. One can see that at fixed target energies the values of $\alpha$ are slightly higher than unity, which is not in contradiction with the result of Ref. [1]. However $\alpha$ decreases with increasing energy and this effect is larger in the case of charm production than in the case of beauty. One can see also that the difference between two variants for $R_{b}^{A}\left(x, \mu^{2}\right)$ at $x<10^{-3}$ becomes important only at the highest energies.

We also calculate the values of $\alpha$ for different Feynman- $x\left(x_{F}\right)$ regions using $x_{F}=$ $x_{a}-x_{b}$ at energies $\sqrt{s_{N N}}=39 \mathrm{GeV}$ and $1800 \mathrm{GeV}$. The results are presented in Fig. 3. At negative and moderate $x_{F}$ (in the nucleus fragmentation region) the values of $\alpha$ are slightly higher than unity. However in the case of charm production in the beam fragmentation region (positive $x_{F}$ ) the values of $\alpha$ become essentially smaller than unity. For beauty production the last effect is expected only at very high energies.

\footnotetext{
${ }^{1}$ Let us note that the theoretical estimations in an earlier paper 12] give more or less a similar behaviour of $R_{b}^{A}\left(x, \mu^{2}\right)$ for light sea quarks but an enhancement of gluon distributions in the nuclei at small $x$.
} 


\section{Conclusions}

We calculate the A-dependence of charm and beauty production using standard QCD formulas and accounting in the difference of parton distributions for free and bound nucleons. If one parametrize the heavy flavour production cross section as $\sigma \sim A^{\alpha}$, the value of $\alpha$ is slightly different from unity at the available energies. At comparatively low energies the obtained values of $\alpha$ are a little larger than unity. This should be connected with some nucleon-nucleon correlations which change the large- $x$ parton distributions.

At higher energies the values of $\alpha$ decrease and become smaller than unity. At $\sqrt{s_{N N}}$ $=1800 \mathrm{GeV}$ we expect the value of $\alpha \sim 0.95$. The decrease of the ratios $R_{b}^{A}\left(x, \mu^{2}\right)$ that results in a decrease of $\alpha$ can be connected with the effects of parton density saturation [18 which occurs in heavy nuclei at $x$ values higher than in the proton.

If we consider two small and different values of $x_{a}$ and $x_{b}$ in Eq. (1), it is clear that the contribution to the inclusive cross section from the region $x_{a}<x_{b}$ should be larger than the mirrow contribution $\left(x_{a}>x_{b}\right)$ because the value of the ratio $R_{b}^{A}\left(x, \mu^{2}\right)$ in the first case is larger. It means that heavy quark pairs will be produced preferably in the nucleus fragmentation hemisphere, i.e., asymetrically, that is quite usual and confirmed experimentally in the case of light quark hadron production. From Fig. 3 it is clear that charm production on nucleus at LHC energies should give information on the nuclear shadowing of the structure functions at small $x$.

Let us note also that at HERA-B energy $\left(\sqrt{s_{N N}}=39 \mathrm{GeV}\right)$ with $\mathrm{GRV}(\mathrm{HO})$ parton distributions we obtain $\sigma(p p \rightarrow b \bar{b})=6.8 \mathrm{nb}$ that is in agreement with the experimental value $5.7 \pm 1.5 \pm 1.3 \mathrm{nb}$ of Ref. [19]. However the experimental point should be decreased by $5 \%$ because it was obtained as an extrapolation from the gold target data assuming an $A^{1}$ dependence whereas our calculations give the value $\alpha=1.01$.

We are grateful to M.A.Braun for useful discussions and both to K.J.Eskola and to I.Sarcevic for sending us their numerical results. We thank the Dirección General de Política Científica and the CICYT of Spain for financial support. N.A. and C.A.S. also thank the Xunta de Galicia for financial support. The paper was also supported in part by INTAS grant 93-0079. 
Table 1

Perturbative QCD predictions for the $\alpha$ values of charm and beauty production in high energy $p A$ interactions with gluon distribution ratios frozen at $x=10^{-3}$.

\begin{tabular}{|c||r|r|r|r|r|r|}
\hline \multicolumn{1}{|c||}{$\sqrt{s}$, GeV } & \multicolumn{2}{c|}{ MSR-1 } & \multicolumn{2}{c|}{ MT S-DIS } & \multicolumn{2}{c|}{ GRV(HO $)$} \\
\cline { 2 - 7 } & $c \bar{c}$ & $b \bar{b}$ & $c \bar{c}$ & $b \bar{b}$ & $c \bar{c}$ & $b \bar{b}$ \\
\hline 27 & 1.01 & 1.01 & 1.01 & 1.00 & 1.01 & 1.01 \\
39 & 1.01 & 1.01 & 1.01 & 1.01 & 1.01 & 1.01 \\
62 & 1.00 & 1.01 & 1.00 & 1.01 & 1.00 & 1.01 \\
120 & 0.99 & 1.01 & 0.99 & 1.01 & 0.99 & 1.01 \\
200 & 0.99 & 1.01 & 0.99 & 1.01 & 0.98 & 1.00 \\
1800 & 0.96 & 0.99 & 0.96 & 0.99 & 0.95 & 0.99 \\
14000 & 0.94 & 0.98 & 0.94 & 0.98 & 0.93 & 0.97 \\
\hline
\end{tabular}




\section{Figure captions}

Fig. 1 : The functions $R_{G}^{A}\left(x, \mu^{2}\right), R_{V}^{A}\left(x, \mu^{2}\right)$ and $R_{S}^{A}\left(x, \mu^{2}\right)$ which determine the ratios of the distributions for protons in the nucleus versus free protons for gluons (solid and dash-dotted curves, see text), valence quarks (dashed curves) and sea quarks (dotted curves) respectively for $\mu^{2}=4 \mathrm{GeV}^{2}$ (a) and $\mu^{2}=m_{b}^{2}$ (b).

Fig. 2 : The energy dependence of $\alpha$ in the cases of charm and beauty production for GRV HO structure functions and using extrapolated (solid curves) and frozen at $x=10^{-3}$ (dashed curves) ratios of gluon distributions.

Fig. 3 : The Feynman- $x$ dependence of $\alpha$ values in the cases of charm and beauty production at $\sqrt{s_{N N}}=39 \mathrm{GeV}$ (a) and $1800 \mathrm{GeV}$ (b) for GRV HO structure functions and using extrapolated (solid curves) and frozen at $x=10^{-3}$ (dashed curves) ratios of gluon distributions. 


\section{References}

[1] M.J.Leitch et al. Phys.Rev.Lett. 72 (1994) 2542.

[2] E.M. Coll., J.J.Aubert et al. Phys.Lett. B123 (1983) 275.

[3] A.Bodek et al. Phys.Rev.Lett. 50 (1983) 1431; 51 (1983) 534.

[4] R.G.Arnold et al. Phys.Rev.Lett. 52 (1984) 727.

[5] BCDMS Coll., G.Bari et al. Phys.Lett. B163 (1985) 282.

[6] EM Coll., J.Ashman et al. Phys.Lett. B202 (1988) 603; M.Arneodo et al. Phys.Lett. B211 (1988) 493.

[7] EM Coll., M.Arneodo et al. Nucl.Phys. B333 (1990) 1.

[8] NM Coll., P.Amaudruz et al. Z.Phys. C51 (1991) 387.

[9] K.J.Eskola. Nucl.Phys. B400 (1994) 240.

[10] P.Nason, S.Dawson and R.K.Ellis. Nucl.Phys. B303 (1988) 607.

[11] I.Sarcevic and P.Valerio. Phys.Lett. B338 (1994) 426.

[12] V.Barone, M.Genovese, N.N.Nikolaev, E.Predazzi and B.G.Zakharov. Z.Phys. C58 (1993) 451.

[13] M.B.Gay Ducati, A.L.Ayala and E.M.Levin. Preprint CBPF-NF-010/95, hep$\mathrm{ph} / 9506349$.

[14] A.D.Martin, R.G.Roberts and W.J.Stirling. Phys.Rev.D37 (1988) 1161.

[15] J.G.Morfin and W.-K.Tung. Z.Phys. C52 (1991) 13.

[16] M.Gluck, E.Reya and A.Vogt. Z.Phys. C53 (1992) 127.

[17] PDFLIB: Nucleon, Pion and Photon Parton Density Functions and $\alpha_{s}$ Calculations, version 6.06. H. Plothow-Besch, CERN-PPE (1995).

[18] L.V.Gribov, E.M.Levin and M.G.Ryskin. Phys.Rep. 100 (1983) 1.

[19] D.M.Jansen et al. Phys.Rev.Lett. 74 (1995) 3118. 


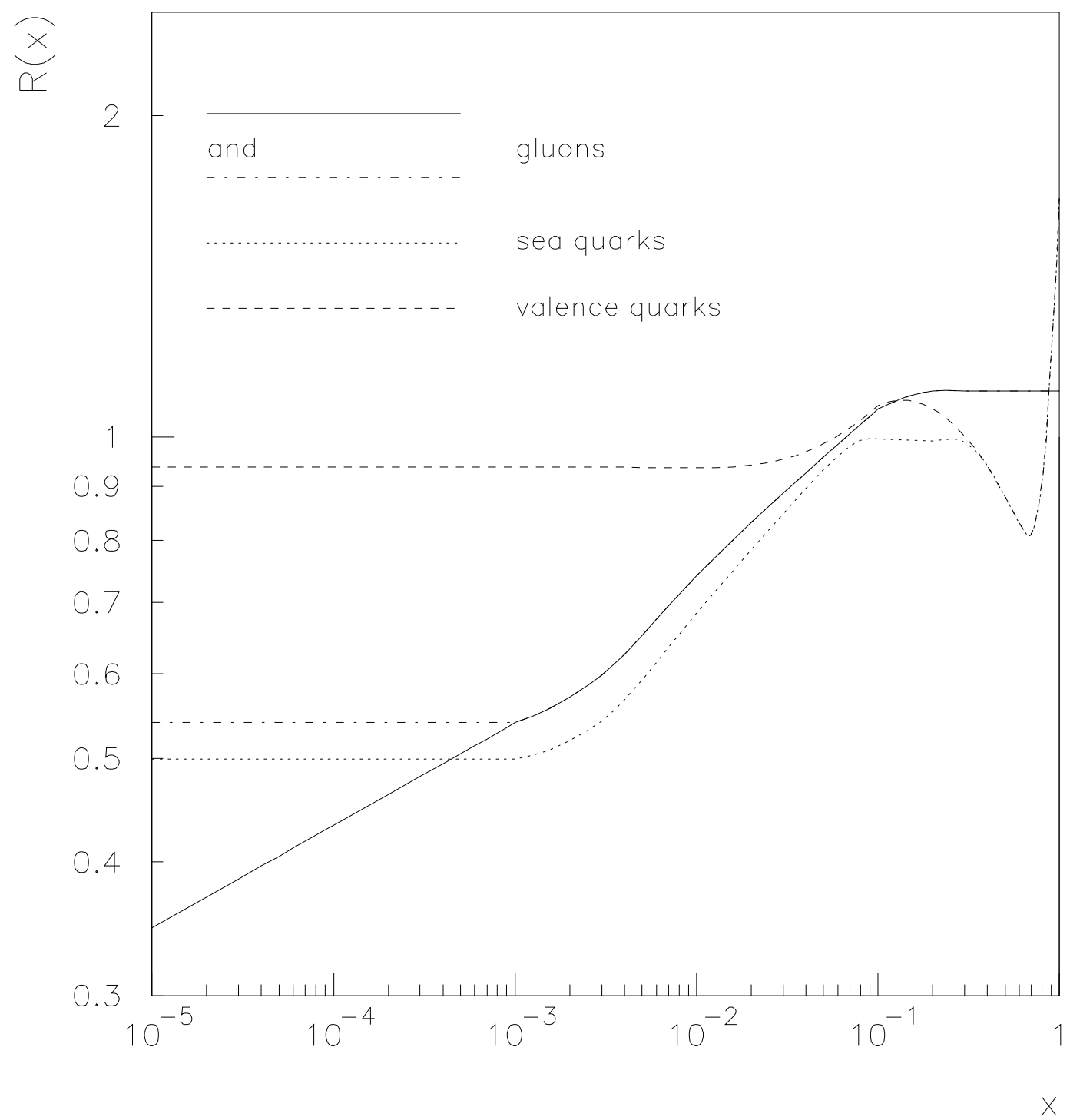

Fig. 1a 


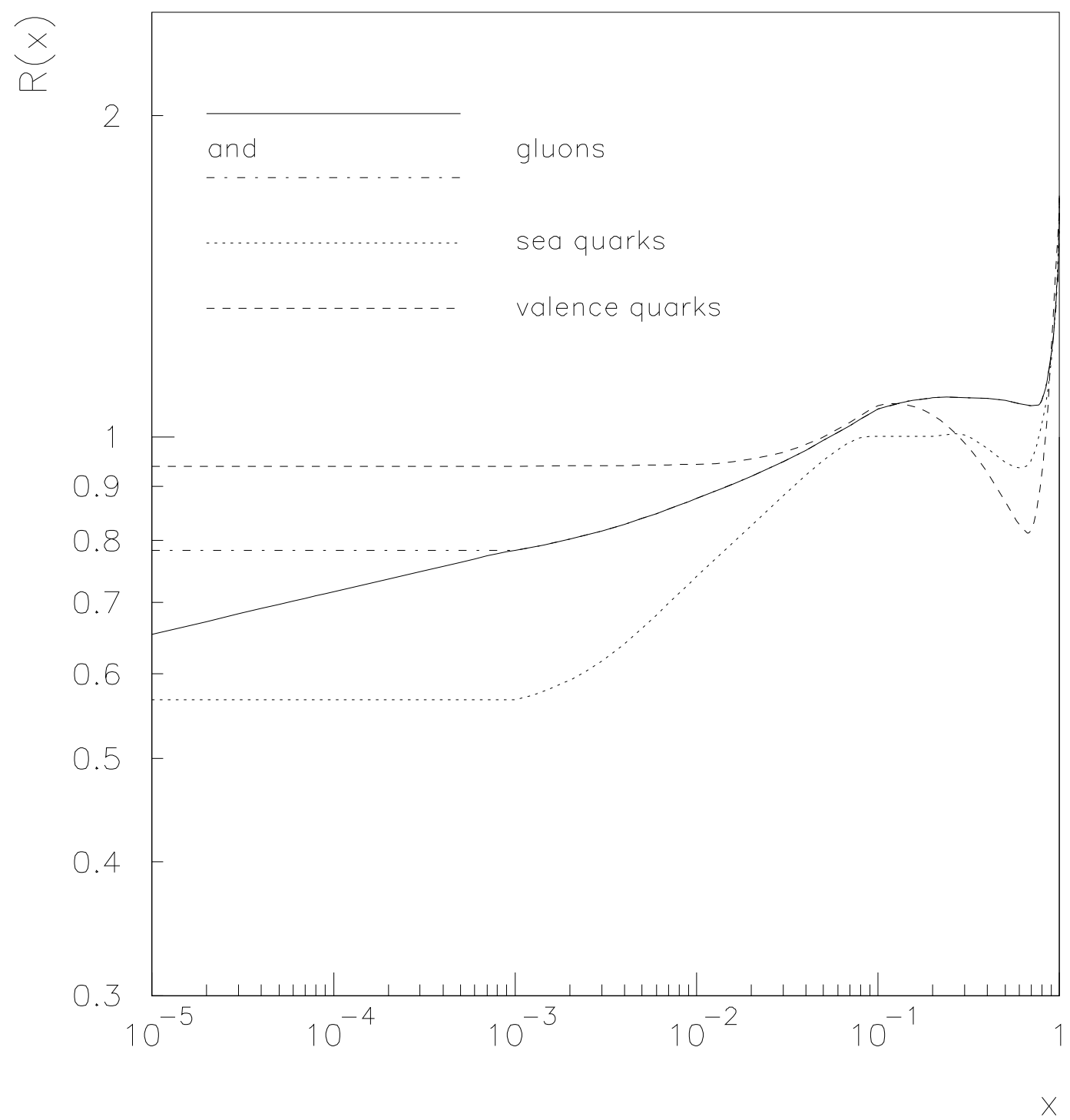

Fig. $1 b$ 


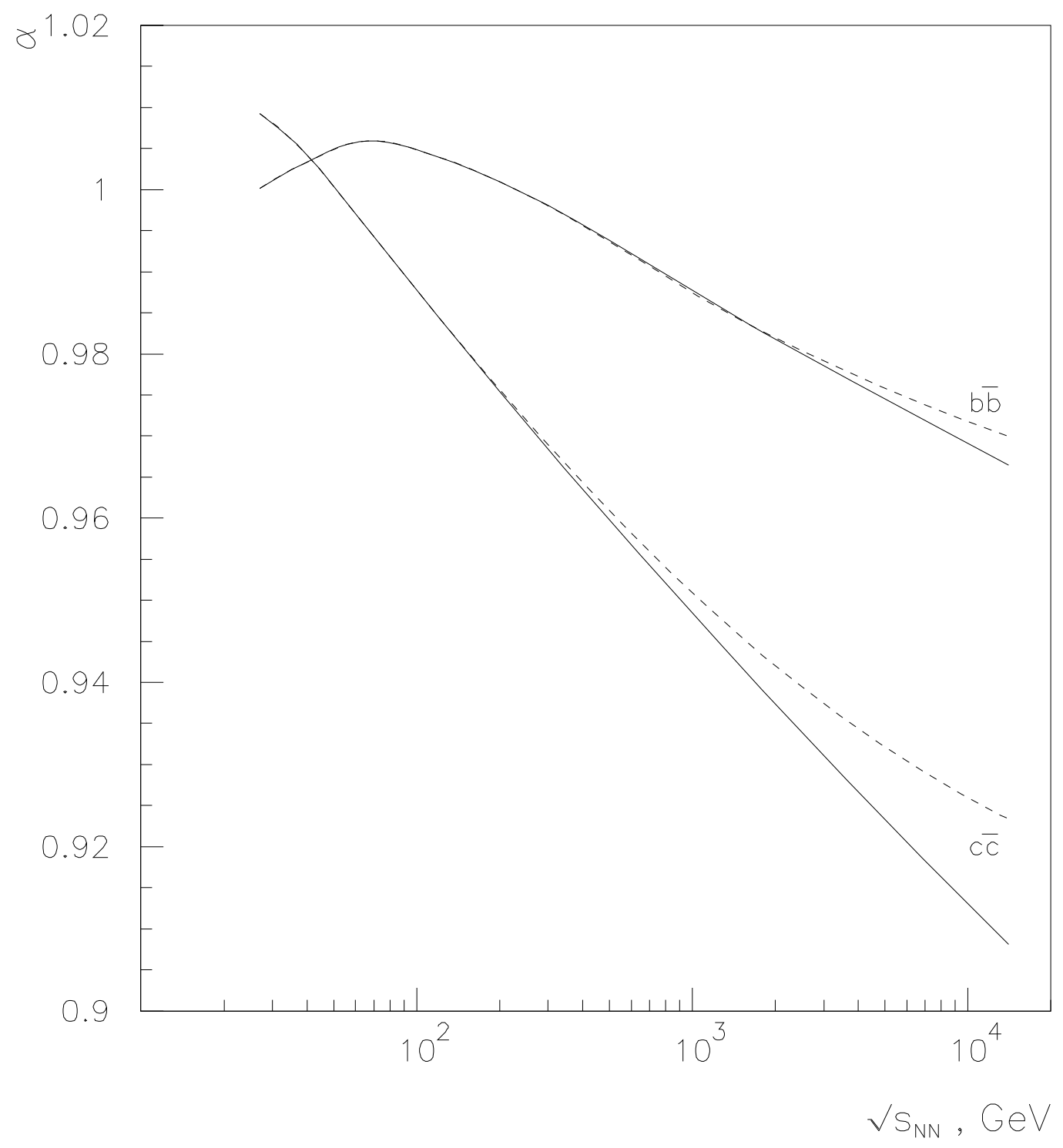

Fig. 2 


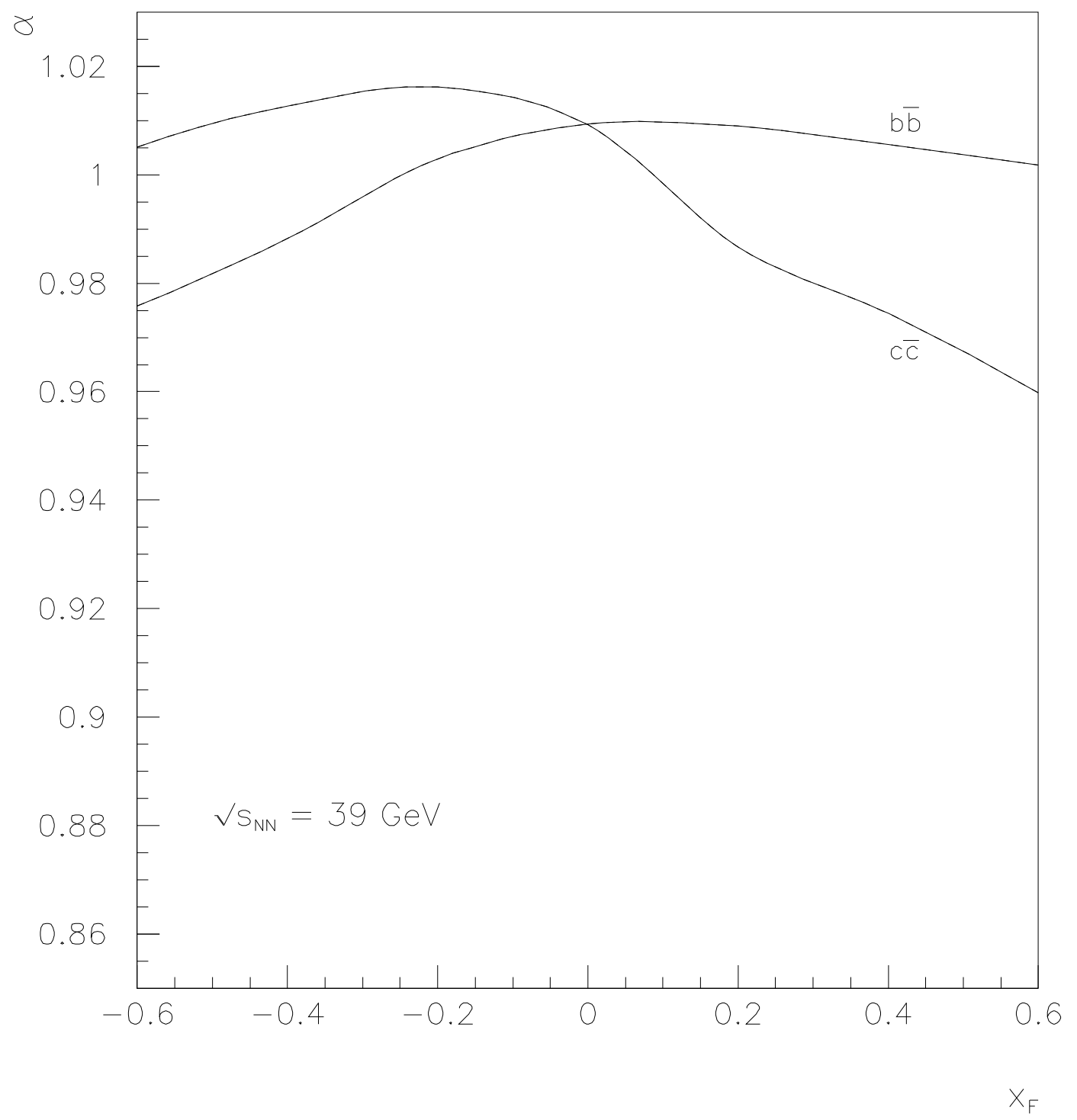

Fig. $3 a$ 


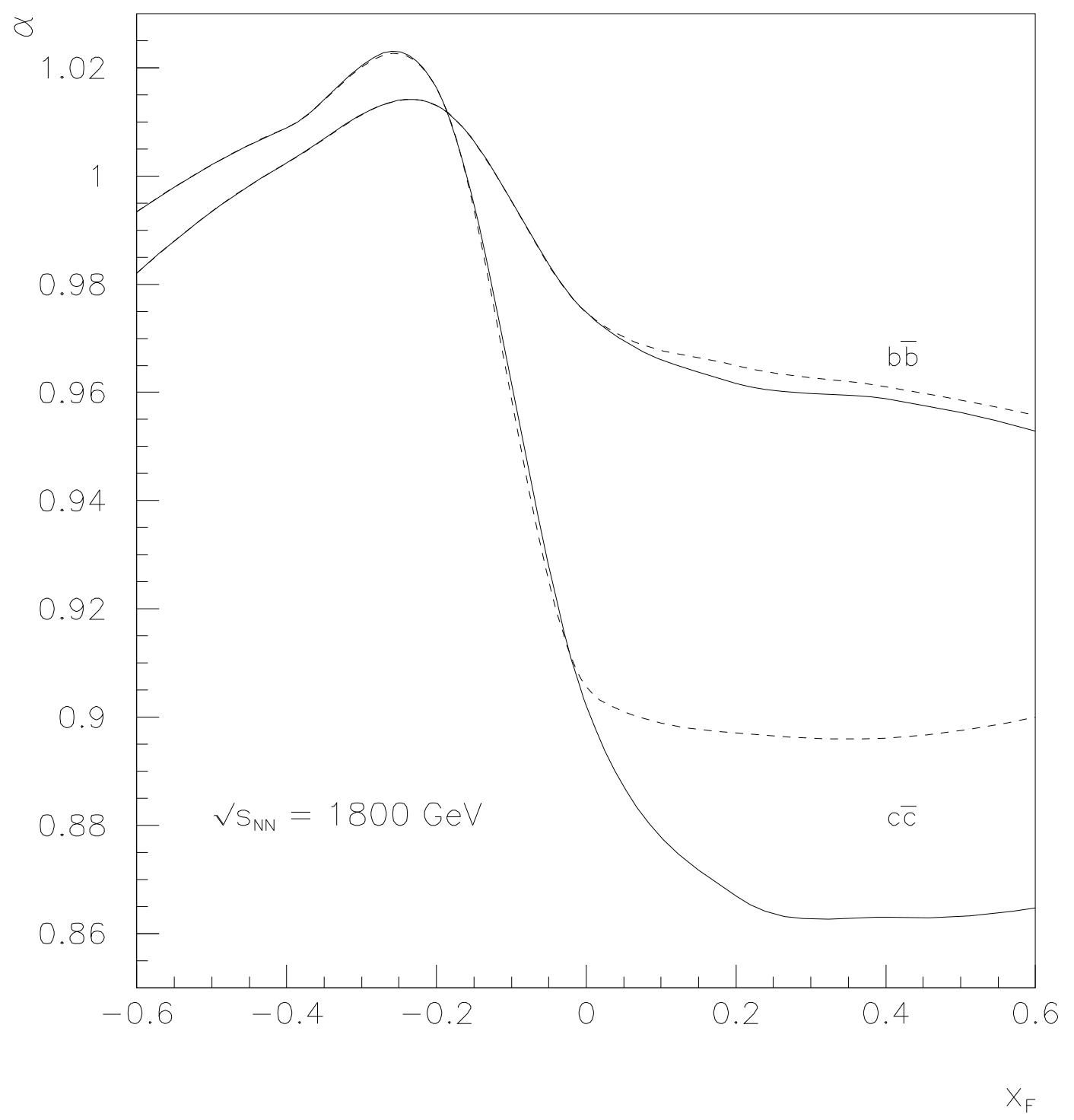

Fig. 3b 